\title{
Warnings as a directive front-of-pack nutrition labelling scheme: comparison with the Guideline Daily Amount and traffic-light systems
}

\author{
Alejandra Arrúa ${ }^{1}$, Leandro Machín ${ }^{1}$, María Rosa Curutchet ${ }^{2}$, Joseline Martínez ${ }^{2}$, \\ Lucía Antúnez ${ }^{3}$, Florencia Alcaire ${ }^{3}$, Ana Giménez ${ }^{3}$ and Gastón Ares ${ }^{1,3, *}$ \\ 'Centro de Investigación Básica en Psicología (CIBPsi), Facultad de Psicología, Universidad de la República \\ (UdelaR), Tristán Narvaja 1674, Montevideo, Uruguay: ${ }^{2}$ Instituto Nacional de Alimentación, Piedras, Montevideo, \\ Uruguay: ${ }^{3}$ Sensometrics \& Consumer Science, Instituto Polo Tecnológico de Pando, Facultad de Química, \\ Universidad de la República, Pando, Canelones, Uruguay
}

Submitted 9 February 2017: Final revision received 30 March 2017: Accepted 7 April 2017: First published online 19 June 2017

\begin{abstract}
Objective: Warnings have recently been proposed as a new type of directive front-of-pack (FOP) nutrition labelling scheme to flag products with high content of key nutrients. In the present work, this system was compared with the two most common FOP nutrition labelling schemes (Guideline Daily Amounts (GDA) and traffic-light system) in terms of goal-directed attention, influence on perceived healthfulness and ability to differentiate between products.

Design/Setting/Subjects: Goal-directed attention to FOP labels was evaluated using a visual search task in which participants were presented with labels on a computer screen and were asked to indicate whether labels with high sodium content were present or absent. A survey with 387 participants was also carried out, in which the influence of FOP labels on perceived healthfulness and ability to identify the healthful alternative were evaluated.

Results: Warnings improved consumers' ability to correctly identify a product with high content of a key nutrient within a set of labels compared with GDA and received the highest goal-directed attention. In addition, products with high energy, saturated fat, sugar and/or sodium content that featured warnings on the label were perceived as less healthful than those featuring the GDA or traffic-light system. Warnings and the traffic-light system performed equally well in the identification of the most healthful product.

Conclusions: Results from the present work suggest that warnings have potential as directive FOP nutrition labels to improve consumer ability to identify unhealthful products and highlight advantages compared with the traffic-light system.
\end{abstract}

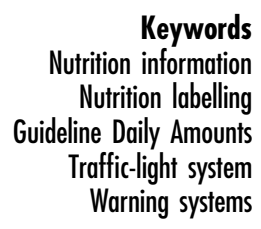

Increasing consumption of ultra-processed products with high energy, fat, sodium and sugar content has been identified as one of the causes of the growing prevalence rates of obesity and other non-communicable diseases ${ }^{(1,2)}$. For this reason, public policies aimed at encouraging consumers to change their eating patterns have been deemed necessary to cope with the increasing public health, economic and social costs associated with these diseases $^{(3,4)}$

Nutrition labels have received considerable attention in the last decade as a tool to empower consumers to evaluate the healthfulness of packaged products and to encourage more healthful food choices ${ }^{(5,6)}$. However, conventional nutrition labels have failed to achieve their goal due to their complexity and their location on the back or the side of packages ${ }^{(7-9)}$.
Front-of-pack (FOP) nutrition labels have been suggested as an effective alternative to improve consumers' ability to find and use nutritional information ${ }^{(10)}$. Different formats of FOP labels have been developed, which can be classified according to the degree to which they assist consumers to draw conclusions on product healthfulness ${ }^{(11)}$. Non-directive systems, such as the Guideline Daily Amount (GDA) system, only provide information about nutrient content in absolute value (per portion or $100 \mathrm{~g}$ ) or as the percentage of the recommended daily intake. Semi-directive systems also communicate information about the content of key nutrients but, in addition, indicate whether they are low, medium or high. The traffic-light system, the most common semi-directive system, uses traffic lights' colour coding (red, yellow and green) as an interpretational aid to classify nutrient 
content $^{(12)}$. Finally, directive systems do not convey information about nutrient content but highlight products that meet specific nutritional criteria. The most common directive systems are health logos, such as the Nordic Keyhole $^{(13)}$, the Australian Health Star rating system $^{(14)}$, Smart Choice ${ }^{(15)}$ or Pick the Tick ${ }^{(16)}$, which highlight healthful products based on predefined limits for specific nutrients.

Research has shown that, compared with non-directive systems, directive and semi-directive systems improve consumers' ability to find and understand nutritional information ${ }^{(17-20)}$. Consumers tend to prefer simple FOP labels and to appreciate interpretational aids, such as the text descriptors or colour codes ${ }^{(10,11,21)}$. Consumers have been reported to find it difficult to make healthfulness judgements based on several nutrients simultaneously ${ }^{(22)}$, suggesting that simple labels that provide an overall evaluation of product healthfulness can improve consumers' ability to differentiate between healthful and unhealthful products. However, health logos have been reported to be likely to increase perceived healthfulness of healthful products rather than to decrease perceived healthfulness of unhealthful products ${ }^{(19)}$. This suggests that positive directive FOP labels may not be sufficient to discourage consumption of unhealthful products.

In this context, warnings have recently been proposed as a new type of directive FOP nutrition label to flag products with high content of key nutrients ${ }^{(23)}$. This system has been implemented recently in Chile and recommended by the Pan American Health Organization to discourage consumption of ultra-processed products $^{(24,25)}$. According to Chilean legislation, products with high energy, sodium, sugar and/or saturated fat content should include separate stop-sign-shaped black warning labels for each nutrient on the front of the $\mathrm{pack}^{(24)}$. However, no study has been published yet evaluating its potential to communicate product healthfulness or comparing its performance with other FOP labels.

The efficiency of FOP labels depends on the extent to which they improve consumers' ability to detect, read and understand nutritional information ${ }^{(26,27)}$. Attention to FOP labels is a prerequisite for information processing that leads to informed food choices ${ }^{(26)}$. Therefore, the more salient FOP labels are within packages and the easier it is to find relevant information, the more likely consumers are to notice them and, consequently, to take them into account in their decision-making process ${ }^{(28)}$. Several studies have used different methodologies, such as visual search, change detection and eye-tracking, to evaluate goal-directed attention to FOP labels when consumers have explicit nutrition goals (e.g. finding a label with low sodium content) and attentional capture when consumers are not explicitly asked to look for nutrition information (e.g. in a shopping scenario) ${ }^{(17,28-30)}$. Once FOP labels are attended to, understanding is a key determinant of whether consumers' food choices can be influenced by nutritional information ${ }^{(26)}$. For this reason, most recent studies have focused on consumers' attention to and understanding of nutrition labels to provide information to stakeholders on which is the most efficient FOP nutrition labelling scheme $e^{(8,28-33)}$.

The aim of the present work was to compare warnings with the two most common non-directive and semidirective FOP nutrition labels (GDA and traffic-light system, respectively) in terms of goal-directed attention, influence on perceived healthfulness of products with high energy, sugar, fat and/or sodium content, and ability to differentiate between healthful and less healthful products.

\section{Materials and methods}

Two studies were conducted to compare warnings with the GDA and traffic-light systems. In a first study, goaldirected attention to FOP labels was evaluated using a visual search task in which participants were presented with labels on a computer screen and were asked to indicate whether labels with high sodium content were present or absent ${ }^{(34)}$. In the second study, a survey with 387 participants was carried out in which the influence of FOP labels on perceived healthfulness and ability to identify the healthful alternative were evaluated. The studies were approved by the Ethics Committee of the School of Chemistry of Universidad de la República (Uruguay).

\section{Front-of-pack nutrition labelling schemes}

Three nutrition labelling schemes were considered: the GDA system, the traffic-light system and the Chilean warning system. The three schemes contained information about energy, sugars, saturated fat and sodium. The trafficlight system classified nutrient content as low, medium or high using text descriptors and colour codes (green, yellow and red, respectively), whereas the Chilean warning system indicated only high nutrient content using separate black stop signs for each nutrient. The traffic-light system included text descriptors and colour for energy content only when it was high. Chilean regulations were used to classify nutrient content as high, whereas the criteria of the UK Food Standards Agency were considered to classify nutrient content as low ${ }^{(12,24)}$.

\section{Visual search study}

\section{Participants}

A total of thirty-two people (fourteen males and eighteen females, age ranging from 18 to 50 years) participated in the study. Participants were recruited from the consumer database of the research group who authored the study, based on their interest and availability to participate. All participants had normal or corrected-to-normal vision and full colour vision. 


\section{Stimuli}

The stimuli presented to participants were sets of labels with FOP nutritional information. Three independent variables were considered for label design: (i) product category (chicken nuggets, hamburgers and instant soup); (ii) sodium content (medium and high); and (iii) FOP nutrition labelling scheme (GDA, traffic-light and warning systems). The three selected products differed in their nutritional composition. Instant soup labels had low content of sugar and saturated fat and medium/high sodium content. Meanwhile, hamburgers and chicken nuggets had low sugar content, high content of saturated fat and medium/high sodium content. This was reflected in the traffic-light system and the warning system: labels of hamburgers and chicken nuggets with high sodium content also had high saturated fat content highlighted using red colour and a separate warning sign, respectively.

Labels were designed using GIMP 2.6 software (Free Software Foundation, Boston, MA, USA) and did not correspond to products currently available in the market to avoid the influence of any previous experience with the products. Nutritional information was included on the labels using the FOP labelling schemes previously described.

For each type of FOP label and product, two types of sets of three labels were created according to the presence of a label with high salt content within the set (present $v$. absent). Within each set, the nutritional composition of the labels and their position on the screen were randomized to avoid consumers' familiarization during the task. The nutritional composition of the labels with medium and high sodium content for each product category is shown in Table 1. An example of one of the sets of chicken nugget labels evaluated in the study is presented in Fig. 1.

\section{Procedure}

Following standard practice in visual search tasks ${ }^{(34)}$, participants performed two training sessions before conducting the test to familiarize them with the experimental procedure and reduce their individual variability. Each training session included two tests of ninety-eight trials, which were not related to food labels to avoid any potential influence on the results. The tasks involved geometrical figures, in which squares were the distractors and a circle the target. Participants were asked to indicate if the target was on the screen or not.

Once training sessions were completed, participants were asked to run the task. The task was organized in three blocks of trials, one for each FOP nutrition label. Blocking of trials by FOP labelling scheme was used to simulate what would happen if they were implemented: consumers would have to compare products with the same scheme. This allowed participants to use scheme-specific strategies to identify labels with high content of a target nutrient during the visual search task. Within a block, sixty trials were presented to respondents, six dummy trials aimed at familiarizing participants with the task and fiftyfour trials of the experimental design. Dummy trials were excluded from further analysis. Before starting the test, respondents were instructed that the term 'high sodium content' corresponded to labels with a sodium content equal or higher than $400 \mathrm{mg}$ per portion and were shown examples of each nutrition labelling scheme.

Trials were presented on a personal computer screen following a balanced rotation (Williams' latin square) using DirectRT software ${ }^{(35)}$. Trials were displayed (one by one) until a key was pressed to respond. Participants were asked to indicated if any label was high in sodium by pressing the S key (for 'si', yes in Spanish) or not, by pressing the $\mathrm{N}$ key (for 'no'). Participants were asked to respond as quickly and accurately as possible, and their responses and response times were recorded by the software. After each block respondents were encouraged to take a $5 \mathrm{~min}$ break before completing the following task.

\section{Data analysis}

Participants' response time and type of response (true $v$. false) were registered. Consumers' responses were classified using a binary coding system; true if the participant correctly classified the label, and false otherwise. A logistic regression was used to evaluate the influence of nutrition labelling scheme on the probability of consumers correctly indicating the existence of a label with high sodium content in the series. A significance level of $5 \%$ was used as the criterion for statistical significance.

Table 1 Nutritional composition of the labels included in the visual search task, in which participants had to evaluate sets of three labels and indicate if there was a label with high sodium content; and the levels of the nutrients classified as low, medium and high*

\begin{tabular}{|c|c|c|c|c|c|c|c|}
\hline \multirow{3}{*}{$\frac{\text { Product category }}{\text { Hamburgers }}$} & \multirow{3}{*}{$\begin{array}{c}\text { Energy (kcal/portion) } \\
184-220 \\
194-200\end{array}$} & \multicolumn{2}{|c|}{ Sugar (g/portion) } & \multicolumn{2}{|c|}{ Saturated fat (g/portion) } & \multicolumn{2}{|c|}{ Sodium (mg/portion) } \\
\hline & & $0-0.5$ & Low & $6 \cdot 5-7 \cdot 1$ & High & $440-520$ & High \\
\hline & & $0-0.2$ & Low & $6 \cdot 3-6 \cdot 7$ & High & $272-380$ & Medium \\
\hline \multirow[t]{2}{*}{ Chicken nuggets } & $109-185$ & $0.4-1.0$ & Low & $5 \cdot 6-7 \cdot 1$ & High & $450-465$ & High \\
\hline & $170-180$ & $0.3-0.9$ & Low & $5 \cdot 0-6 \cdot 3$ & High & $205-225$ & Medium \\
\hline \multirow{2}{*}{ Instant soup } & $52-62$ & $0.5-2.8$ & Low & $0-0.3$ & Low & $400-510$ & High \\
\hline & $60-70$ & $0.2-2 \cdot 0$ & Low & $0-0.3$ & Low & $65-150$ & Medium \\
\hline
\end{tabular}

*Labels featuring the traffic-light system highlighted the nutrient content shown in the table, whereas labels featuring the warning system included separate warnings for all nutrients with high content. 

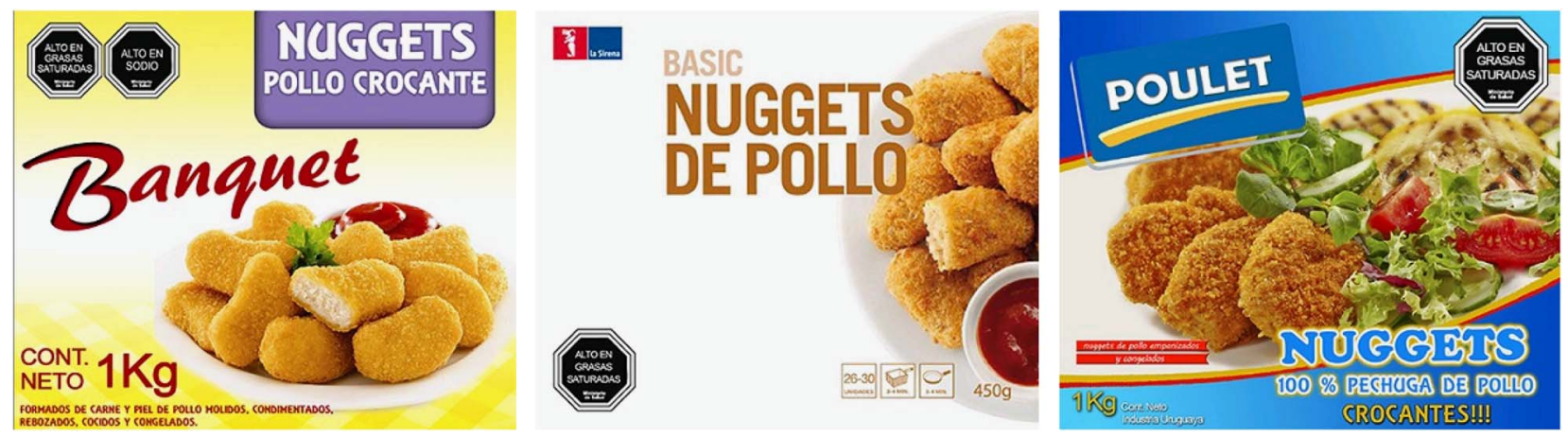

Fig. 1 Example of a set of three chicken nuggets labels presented in the visual search study to evaluate attentional capture of front-of-pack nutrition labelling schemes. The first label contains warnings for high saturated fat and sodium content, whereas the remaining two only contain a warning for high saturated fat content

Response times associated with incorrect answers, as well as response times shorter or longer than three standard derivations from the mean of the experimental condition, were not considered for further analysis. Six different cut-offs were considered, one for each FOP nutrition labelling scheme and type of task (with and without a label with high sodium content in the series). The percentage of trials that were removed prior to the analysis for deviating from the mean corresponded to $1.9 \%$, and ranged between 1.4 and $2.9 \%$ for the six individual experimental conditions.

The influence of the experiment variables (type of product, nutrition label scheme and presence of a label with high sodium content in the series) and their interaction on reaction times was evaluated using a repeatedmeasures ANOVA. When the effects were significant, least significant differences were calculated using Fisher's test at a significance level of $5 \%$.

All statistical analyses were performed using $R$ language ${ }^{(36)}$.

\section{Healthfulness perception}

\section{Participants}

Three hundred and eighty-seven people (age ranging between 18 and 84 years, $67 \%$ females) were recruited from public places in the cities of Montevideo and Flores (Uruguay). The characteristics of the participants, in terms of age, gender, educational and socio-economic level, are shown in Table 2.

\section{Experimental procedure}

Participants were randomly allocated to one of three experimental conditions, each of which corresponded to one of the FOP labelling schemes (GDA, traffic-light and warning systems). Thus, each participant evaluated labels featuring one of the FOP schemes. The number of participants in each group was: 136 (warning system), 127 (GDA system) and 124 (traffic-light system). No significant differences in gender, age, educational and socioeconomic level distribution of the three groups of
Table 2 Sociodemographic characteristics of Uruguayan adult participants in the healthfulness evaluation study $(n 387)$

\begin{tabular}{lr}
\hline Characteristic & $\%$ \\
\hline Gender & \\
Female & 68 \\
Male & 32 \\
Age (years) & \\
$18-25$ & 15 \\
$26-35$ & 37 \\
$36-45$ & 28 \\
$46-60$ & 15 \\
$>60$ & 5 \\
Educational level & 4 \\
Primary school & 59 \\
Secondary school & 18 \\
Technical education & 16 \\
University degree & 3 \\
Postgraduate studies & \\
Socio-economic level & 21 \\
Low & 59 \\
Medium & 20 \\
High & \\
\hline
\end{tabular}

participants who evaluated different FOP nutrition labelling schemes were found $(P>0 \cdot 26)$. Participants were instructed that they had to evaluate the healthfulness of a set of labels using their own criteria in two different tasks, which are explained below.

\section{Healthfulness evaluation}

Participants received five labels of different product categories (breakfast cereals, crackers, frozen lasagne, instant soup and pan bread), one by one, following an experimental design balanced for presentation and carry-over effects (Williams' latin square). Labels did not correspond to products available in the marketplace and contained a high content of at least one key nutrient (energy, saturated fat, sugar and sodium). The nutritional composition included on the labels was similar to that of commercial products and is shown in Table 3.

For each label, participants had to rate their perceived healthfulness using a 7-point scale ( $1=$ 'not healthful', $7=$ 'very healthful') and the frequency with which they should consume it using a 5-point scale (1 = 'I should 
Table 3 Nutritional composition of the labels included in the tasks in which participants had to select the most healthful product and to evaluate product healthfulness; and the levels of the nutrients classified as low, medium and high*

\begin{tabular}{|c|c|c|c|c|c|c|c|c|c|}
\hline \multirow{2}{*}{$\frac{\text { Product category }}{\text { Breakfast cereals }}$} & \multirow{2}{*}{$\frac{\text { Alternative }}{1 \dagger}$} & \multicolumn{2}{|c|}{ Energy (kcal/portion) } & \multicolumn{2}{|c|}{ Sugar (g/portion) } & \multicolumn{2}{|c|}{ Saturated fat (g/portion) } & \multicolumn{2}{|c|}{ Sodium (mg/portion) } \\
\hline & & 337 & High & $9 \cdot 3$ & HIgh & 0 & Low & 130 & Low \\
\hline & 2 & & & 4.0 & High & 0 & Low & 150 & Low \\
\hline \multirow[t]{3}{*}{ Chicken nuggets } & 1 & & & 1.0 & Low & $5 \cdot 6$ & High & 350 & High \\
\hline & 2 & & & 0.3 & Low & $6 \cdot 3$ & High & 210 & Medium \\
\hline & 3 & & & 0.8 & Low & $5 \cdot 8$ & High & 355 & High \\
\hline \multirow[t]{2}{*}{ Cookies } & 1 & 130 & High & $6 \cdot 7$ & High & 3.9 & High & 24 & Low \\
\hline & 2 & 112 & High & 8.4 & High & 1.3 & Medium & 41 & Low \\
\hline \multirow[t]{3}{*}{ Crackers } & $1 \dagger$ & 128 & High & 0.6 & Low & $2 \cdot 1$ & High & 114 & Medium \\
\hline & 2 & 127 & High & 0.3 & Low & 1.9 & High & 251 & High \\
\hline & 3 & 129 & High & 0.3 & Low & $2 \cdot 0$ & High & 210 & High \\
\hline \multirow{2}{*}{ Fish nuggets } & 1 & & & 1.4 & Low & 1.4 & Medium & 315 & High \\
\hline & 2 & & & 1.6 & Low & 1.5 & Medium & 175 & Medium \\
\hline \multirow[t]{3}{*}{ Frozen hamburgers } & 1 & & & 0 & Low & $6 \cdot 6$ & High & 272 & Medium \\
\hline & 2 & & & 0 & Low & 6.5 & High & 470 & High \\
\hline & 3 & & & 0 & Low & $6 \cdot 7$ & High & 520 & High \\
\hline \multirow[t]{2}{*}{ Frozen lasagne } & 1 & 268 & High & 2.5 & Low & 13 & High & 775 & Medium \\
\hline & $2 \dagger$ & 268 & High & 1.4 & Low & 11 & High & 1265 & High \\
\hline \multirow[t]{3}{*}{ Instant soup } & 1 & & & 1.8 & Low & 0 & Low & 205 & Medium \\
\hline & 2 & & & 0.5 & Low & 0 & Low & 605 & High \\
\hline & $3 \dagger$ & & & 0.7 & Low & 0.3 & Low & 510 & High \\
\hline \multirow[t]{3}{*}{ Pan bread } & 1 & & & 1.6 & Low & 0.6 & Low & 250 & High \\
\hline & $2 \dagger$ & & & $2 \cdot 2$ & Low & 0.5 & Low & 222 & High \\
\hline & 3 & & & $2 \cdot 0$ & Low & 0.5 & Low & 120 & Medium \\
\hline \multirow{2}{*}{ Yoghurt } & 1 & & & 28 & High & 1.4 & Low & 80 & Low \\
\hline & 2 & & & 14 & Medium & 1.8 & Low & 98 & Low \\
\hline
\end{tabular}

The nutrient that defined the most healthful alternative within each set is highlighted in bold.

*Labels featuring the traffic-light system highlighted the nutrient content in the table, whereas labels featuring the warning system included separate warnings for all nutrients with high content. Only high energy content was highlighted.

†Labels included in the healthfulness perception task.

avoid it', 2 = 'occasionally', $3=$ 'several times a month', $4=$ 'several times a week', $5=$ 'several times a day').

\section{Selection of the most bealthful product}

Ten sets of two or three labels of different product categories (breakfast cereals, chicken nuggets, cookies, crackers, fish nuggets, frozen hamburgers, frozen lasagne, instant soup, pan bread and yoghurt) were created to evaluate participants' ability to use FOP labels to compare products and identify the most healthful alternative. All products are frequently consumed in the country and typically contain high content of at least one key nutrient. Labels did not correspond to commercial products available in the marketplace.

The nutritional information of the labels in each set was modified so that one of the labels was more healthful than the others. For each set, one key nutrient was considered target and its content increased by $63-200 \%$ between the healthful alternative and the rest of the labels of the set, so that its content changed from medium to high according to the criteria implemented by the Chilean government ${ }^{(24)}$. The content of all other nutrients was modified by a trivial amount that did not affect their classification in low/ medium/high. Therefore, the target nutrient determined the most healthful option in each set and, consequently, the 'correct' answer. The nutritional composition of the labels in each set and the target nutrients are shown in Table 3. The order of the alternatives within the set was randomized among participants. An example of how the labels were presented to participants is shown in Fig. 2.

Participants received the ten sets one by one, following an experimental design balanced for presentation and carry-over effects (Williams' latin square). For each set of products participants were asked to indicate the most healthful alternative. After the task, participants were asked to answer a sociodemographic questionnaire.

\section{Data analysis}

ANOVA was used to evaluate the influence of FOP labelling scheme on perceived healthfulness and recommended consumption frequency. Product, FOP labelling scheme and their interaction were considered as fixed sources of variation. A $5 \%$ significance level was considered. When differences were significant, Fisher's test was used for post hoc comparison of means.

ANOVA was also used to evaluate differences across FOP labelling schemes in the mean number of sets for which participants correctly identified the most healthful option. A logistic regression analysis was used to evaluate the influence of nutrition label scheme, product and their interaction on the probability of consumers correctly identifying the most healthful product in each series.

All statistical analyses were performed using $\mathrm{R}$ language ${ }^{(36)}$. 

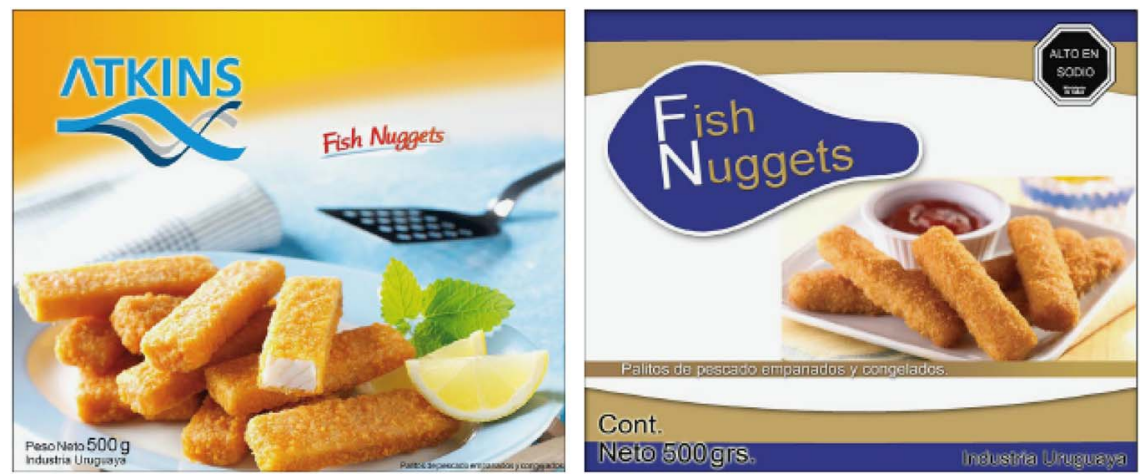

Fig. 2 Example of one of the sets of products considered in the consumer survey to assess participants' ability to use front-of-pack nutrition labelling schemes to compare products and identify the most healthful alternative. The label on the right contains a warning for high sodium content

\section{Results}

\section{Visual search study}

Across all tasks, the average percentage of correct answers was higher than $95 \%$. Logistic regression showed that the percentage of correct answers was significantly lower $(P<0 \cdot 001)$ for the series of labels which contained a label with high sodium content than for series in which none of the labels had high sodium content (94v. 96\%). No significant differences in the percentage of correct responses between products or FOP schemes were found $(P>0.49)$.

Participants' ability to identify a label with high content of a key nutrient was evaluated using response times. Shorter responses times were related to higher ability to find the information. The time needed by participants to respond was significantly shorter $\left(F_{1,31}=116 \cdot 9, \quad P<0.001\right)$ when series included a label with high sodium content in relation to series in which none of the labels had high sodium content (1689 $v$. 1983 ms, respectively). This result can be explained by considering that when none of the labels had high sodium content assessors had to look at all of them to conclude about the absence of labels with high sodium content. On the contrary, when the set included a label with high sodium content, participants could find it without having to look at all of the labels, spending less time to complete the task.

Significant differences in response time between the three FOP nutrition labelling schemes were found $\left(F_{2,62}=36 \cdot 3\right.$, $P<0 \cdot 001)$. The longest response times were observed for series in which nutrition information was presented using the GDA system (mean $2187 \mathrm{~ms}$ ). The inclusion of interpretational aids in the form of directive or semi-directive labels significantly reduced response times. When the trafficlight system was used, the mean response time was reduced to $1784 \mathrm{~ms}$. A further reduction in mean response time to $1422 \mathrm{~ms}$ was achieved by including warning labels.

Type of product also influenced response times $\left(F_{2,62}=45.4, P<0.001\right)$. Participants invested less time when completing the task with instant soup (1671 ms), followed by hamburgers (1797 ms) and finally chicken nuggets (1951 ms). However, differences between products depended on the scheme, as evidenced by the significant interaction $\left(F_{4,124}=2 \cdot 6, P=0 \cdot 04\right)$. In the case of the GDA system, no significant differences were found between the three products, whereas for the traffic-light system and warnings participants needed less time to complete the task for instant soup compared with hamburgers and chicken nuggets. This difference in the time needed to identify the presence of a label with high sodium could be explained by considering the number of nutrients with high content included in the study. Labels of hamburgers and chichen nuggets with high sodium content also had high saturated fat content. Therefore, when interpretative cues (colour or warnings) were included on the labels, participants needed more time to figure out if they corresponded to the target nutrient when compared with instant soup labels, which contained only one nutrient with high content that corresponded to the target nutrient. Differences between hamburguers and chicken nuggets could have been determined by differences in the graphic design of the background on which the FOP labels were included. Label background could have influenced the saliency of the FOP labels and consequently the time needed by participants to find the relevant information to complete the task.

\section{Healthfulness perception}

Healthfulness perception of individual products

Healthfulness perception was significantly influenced by product $\left(F_{4,1903}=36 \cdot 105, P=<0 \cdot 001\right)$ and FOP nutritional information scheme $\left(F_{2,1903}=8.75, P<0.001\right)$, whereas the interaction between product and FOP scheme was not significant $\left(F_{8,1903}=1 \cdot 35, P=0 \cdot 21\right)$. On average, participants in the warning system condition rated product healthfulness significantly lower (mean score 3.6) than participants in both the GDA and the traffic-light system conditions, which did not significantly differ (mean score 4.0).

Table 4 shows mean healthfulness scores for each of the five products considered in the task for the three consumer groups. Frozen lasagne showed the lowest 
Table 4 Mean healthfulness scores for ultra-processed products featuring three different front-of-pack nutritional schemes: Guideline Daily Amounts (GDA), traffic-light system and Chilean warning system

\begin{tabular}{llcc}
\hline Product & $\begin{array}{c}\text { GDA } \\
(n \text { 127) }\end{array}$ & $\begin{array}{c}\text { Traffic-light system } \\
(n \text { 124) }\end{array}$ & $\begin{array}{c}\text { Chilean warning } \\
\text { system }(n \text { 136) }\end{array}$ \\
\hline Frozen lasagne & $3 \cdot 0^{\mathrm{C} ; \mathrm{A}}$ & $3 \cdot 1^{\mathrm{c} ; \mathrm{A}}$ & $3 \cdot 0^{\mathrm{b} ; \mathrm{A}}$ \\
Pan bread & $4 \cdot 0^{\mathrm{b} ; \mathrm{A}}$ & $4 \cdot 2^{\mathrm{a}, \mathrm{b} ; \mathrm{A}}$ & $3 \cdot 7^{\mathrm{a} ; \mathrm{B}}$ \\
Crackers & $3 \cdot 9^{\mathrm{b} ; \mathrm{A}}$ & $3 \cdot 8^{\mathrm{b} ; \mathrm{A}}$ & $3 \cdot 5^{\mathrm{a}, \mathrm{b} ; \mathrm{B}}$ \\
$\begin{array}{l}\text { Breakfast } \\
\text { cereal }\end{array}$ & $4 \cdot 7^{\mathrm{a} ; \mathrm{A}}$ & $4 \cdot 4^{\mathrm{a}, \mathrm{b} ; \mathrm{A}}$ & $4 \cdot 0^{\mathrm{a} ; \mathrm{B}}$ \\
Instant soup & $4 \cdot 2^{\mathrm{b} ; \mathrm{A}, \mathrm{B}}$ & $4 \cdot 5^{\mathrm{a} ; \mathrm{A}}$ & $4 \cdot 0^{\mathrm{a} ; \mathrm{B}}$ \\
\hline
\end{tabular}

${ }^{a, b, c}$ Mean values within a column with unlike lower-case superscript letters were significantly different according to Tukey's test $(P<0.05)$.

$A, B$ Mean values within a row for a product level with unlike upper-case superscript letters were significantly different according to Tukey's test $(P<0.05)$.

healthfulness scores, regardless of the FOP labelling scheme included on the label. For this product, no significant differences between the FOP schemes were found. For pan bread, crackers and breakfast cereals, participants in the warning system condition rated healthfulness significantly lower compared with those in the GDA and the traffic-light system conditions. Meanwhile, healthfulness scores from participants who evaluated products featuring the traffic-light system did not significantly differ from those of participants who evaluated products featuring the GDA system across the five categories.

Consumers' perceived recommended frequency consumption scores were also significantly affected by product $\left(F_{4,1899}=64.41, \quad P<0.001\right)$, FOP scheme $\left(F_{2,1899}=6.85\right.$, $P=0 \cdot 001)$ and their interaction $\left(F_{8,1899}=2 \cdot 13, P=0 \cdot 030\right)$. On average, participants in the warning system condition gave significantly lower scores (mean score 2.7 ) than those in the other two conditions (mean score $2 \cdot 9$ ). As shown in Table 5, participants in the warning system condition rated perceived recommended consumption frequency of two of the five products (crackers and breakfast cereals) significantly lower did than participants in the GDA system. Meanwhile, scores for the traffic-light system did not significantly differ from those of the GDA system for all product categories.

\section{Selection of the most bealthful product}

Significant differences in the number of correct responses were found between the three FOP labelling schemes $\left(F_{2,382}=24 \cdot 64, P<0 \cdot 001\right)$. On average, consumers in the traffic-light and warning system conditions were able to correctly identify the most healthful option in a larger percentage of sets ( 83 and $82 \%$, respectively) compared with those in the GDA condition (67\%). No significant differences between the traffic-light and the warning system were found.

The interaction between FOP schemes and product was significant $(P<0.001)$. As shown in Fig. 3, although differences among FOP schemes on the percentage of
Table 5 Mean perceived recommended consumption frequency scores for ultra-processed products featuring three different frontof-pack nutritional schemes: Guideline Daily Amounts (GDA), trafficlight system and Chilean warning system

\begin{tabular}{lccc}
\hline Product & $\begin{array}{c}\text { GDA } \\
(n \text { 127) }\end{array}$ & $\begin{array}{c}\text { Traffic-light system } \\
(n \text { 124) }\end{array}$ & $\begin{array}{c}\text { Chilean warning } \\
\text { system }(n \text { 136) }\end{array}$ \\
\hline Frozen lasagne & $2 \cdot 0^{\mathrm{C} ; \mathrm{A}}$ & $2 \cdot 1^{\mathrm{b} ; \mathrm{A}}$ & $2 \cdot 2^{\mathrm{b} ; \mathrm{A}}$ \\
Pan bread & $3 \cdot 1^{\mathrm{a} ; \mathrm{A}}$ & $3 \cdot 2^{\mathrm{a} ; \mathrm{A}}$ & $2 \cdot 9^{\mathrm{a} ; \mathrm{A}}$ \\
Crackers & $3 \cdot 1^{\mathrm{a} ; \mathrm{A}}$ & $3 \cdot 1^{\mathrm{a} ; \mathrm{A}}$ & $2 \cdot 8^{\mathrm{a} ; \mathrm{B}}$ \\
Breakfast cereal & $3 \cdot 4^{\mathrm{a} ; \mathrm{A}}$ & $3 \cdot 1^{\mathrm{a} ; \mathrm{A}, \mathrm{B}}$ & $2 \cdot 9^{\mathrm{a} ; \mathrm{B}}$ \\
Instant soup & $2 \cdot 8^{\mathrm{b} ; \mathrm{A}}$ & $2 \cdot 9^{\mathrm{a} ; \mathrm{A}}$ & $2 \cdot 7^{\mathrm{a} ; \mathrm{A}}$ \\
\hline
\end{tabular}

a,b,c Mean values within a column with unlike lower-case superscript letters were significantly different according to Tukey's test $(P<0.05)$.

$A, B$ Mean values within a row for a product level with unlike upper-case superscript letters were significantly different according to Tukey's test $(P<0.05)$.

correct responses depended on the specific product being considered, the traffic-light system and warnings tended to improve consumer ability to identify the most healthful option compared with the GDA system.

\section{Discussion}

Warnings have recently been proposed as a directive FOP nutrition labelling scheme and implemented in Chile to improve consumers' ability to identify products with high content of key nutrients ${ }^{(23,24)}$. In the present work, this new scheme was compared with two of the most widely studied FOP labels, GDA and traffic-light systems, in terms of goal-directed attention, influence on healthfulness perception and ability to differentiate between healthful and less healthful products. Results from the two sub-studies confirmed the potential of the warning system.

In the visual search study, the time needed by participants to detect a label with high sodium content was significantly higher when labels featured the GDA system compared with those featuring the traffic-light system and warnings. This result agrees with Antúnez et al. ${ }^{(17)}$, who reported that interpretational aids improve consumer ability to find and identify labels with high content of a target nutrient. Regarding the comparison of the schemes with interpretational aids, faster performance was observed for warnings compared with the traffic-light system. This can be explained considering that warnings appeared on the labels only when the content of the target nutrient was high, whereas the traffic-light system highlighted high, medium and low nutrient content. Similar results were reported by van Herpen and van Trijp ${ }^{(37)}$ when comparing health logos and the traffic-light system. According to these authors, health logos require less attention time than the traffic-light system, even when there is a time constraint for making a choice between products. Considering that consumers usually invest a few seconds for deciding their food purchases ${ }^{(38)}$ and that 


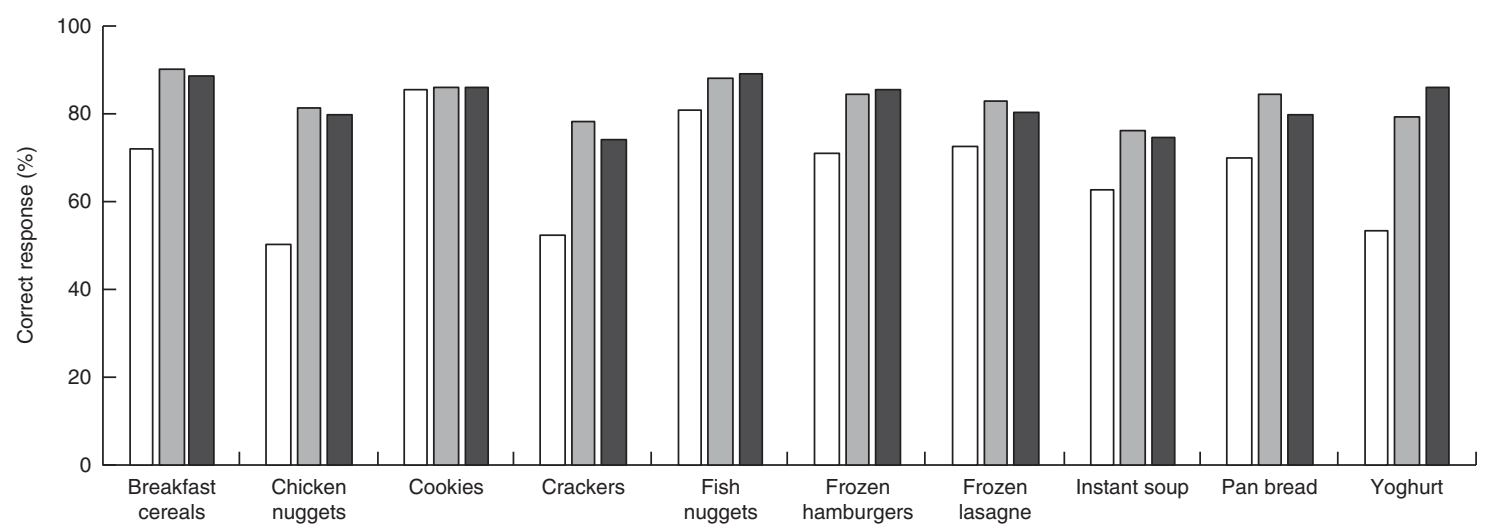

Fig. 3 Percentage of correct responses when participants were asked to evaluate sets of two or three labels and select the most healthful alternative, for three front-of-pack nutrition labelling schemes: Guideline Daily Amounts ( $\square$ ), traffic-light system ( $\square$ ) and Chilean warning system $(\square)$

attention is a prerequisite for information processing ${ }^{(39)}$, results from the visual search study suggest that warnings can improve consumers' ability to identify unhealthful products when making their food choices.

Once consumers acquire nutrition information from FOP labels, understanding plays a key role in determining if the information will influence choice ${ }^{(40)}$. In the present work the influence of the three FOP labels on healthfulness perception and ability to identify the most healthful product was assessed using two separate tasks.

In the first task, products with high energy, saturated fat, sugar and/or sodium content that featured warnings on the label received significantly lower healthfulness scores than those featuring the GDA or the traffic-light system. In addition, warnings encouraged participants to think that two of the five products were less appropriate for frequent consumption. Previous studies have shown that FOP labels that lack reference point information are less effective in communicating product healthfulness when evaluated in isolation ${ }^{(33)}$. Although in the present work warnings were effective in communicating product unhealthfulness, the traffic-light system did not significantly modify healthfulness perception or perceived recommended frequency consumption. This result can be explained by considering that in all products the trafficlight system highlighted low content of at least one nutrient in green, which can convey health associations and may enhance healthfulness perception ${ }^{(41,42)}$. In this sense, a recent study has reported that healthfulness scores of ultra-processed products were significantly lower when featuring a monochromatic traffic-light system compared with a coloured traffic-light system ${ }^{(43)}$. In addition, the traffic-light system required consumers to infer product healthfulness based on the simultaneous evaluation of the level of four nutrients, which is usually a complex task for consumers $^{(22)}$. According to a previous study, although the traffic-light system is usually perceived as friendly, it does not consistently enable differentiation between healthful and less healthful products ${ }^{(19)}$. For this reason, simple directive FOP labels may be more effective than semidirective labels to discourage unhealthful food choices and/or encourage healthful choices.

Consumers' ability to discriminate among products based on their healthfulness was also evaluated. Results showed that both the traffic-light system and warnings improved consumer ability to identify the most healthful alternative. This result is in line with several studies that show that consumers usually find it difficult to assess nutritional information in the form of numbers and percentages ${ }^{(44-46)}$. According to Soederberg Miller et $a l .{ }^{(47)}$, when consumers are not provided with reference information, small differences between products in the content of one or two nutrients may drive the decision, without consideration of other important nutrients. For this reason, interpretational aids and reference information improve consumer ability to identify the most healthful alternative among a set of products ${ }^{(17,19,33,48,49)}$. In the present work, no significant differences were found between warnings and the traffic-light system. Similar results have been reported by Borgmeier and Westenhoefer ${ }^{(48)}$ regarding the comparison of semi-directive and directive FOP labels. Similarly, van Herpen and van Trijp ${ }^{(37)}$ reported that health logos and the traffic-light system did not differ in their ability to stimulate the choice of a healthful product alternative. However, it should be taken into account that warnings may allow a smaller differentiation of products than the traffic-light system as only products with high content of key nutrients are identified. Further research on this respect should be conducted.

In addition to the superiority of warnings over the traffic-light system in terms of goal-directed attention and healthfulness perception of unhealthful products, other considerations related to their implementation should be considered. First of all, traffic lights and warnings differ in the extent to which they inform consumers on the nutrient content of products. Although warnings can improve consumers' ability to identify unhealthful products, they 
do not educate consumers on whether the content of key nutrients is low, medium or high.

Although results confirm the potential of warnings as a directive FOP nutrition labelling scheme, they do not put an end to the debate on which is the best scheme. Further research comparing warnings with the traffic-light system and other directive FOP labels are still necessary to provide stakeholders with objective information to select the system with the largest potential to modify consumer eating patterns. In this sense, it is important to highlight that the present study was not conducted under ecological conditions. It was not carried out in a real purchasing situation, in which consumers face several distractions, and involved the evaluation of a limited number of product categories and alternatives within each set of product. Future studies should explore real or simulated environments and consider the influence of time restriction on consumer perception of nutritional information. In addition, studies exploring the interaction between FOP labels and other package and label elements, such as graphic design and health claims, on consumer healthfulness perception are still necessary.

In closing this section, it is worth highlighting that the efficacy of nutritional information in shifting consumers' eating patterns does not only depend on its ease to be found and understood. In this sense, although several studies have shown that the traffic-light system improves understanding of nutrition information, in-store observations and consumption data have shown a limited effect on behavioural change ${ }^{(48,50-52)}$. However, the inclusion of the traffic-light system or warnings on packages could encourage food companies to reformulate their products to differentiate themselves in the marketplace, which could lead to a reduction in population intakes of sugar, sodium and fat.

\section{Conclusions}

Results from the present work indicate that warnings improved consumer ability to correctly identify a product with high content of a key nutrient within a set of labels compared with the GDA system and received higher goaldirected attention than both the GDA and the traffic-light systems. In addition, products with high energy, saturated fat, sugar and/or sodium content that featured warnings on their labels were perceived as less healthful than those featuring the GDA or traffic-light system.

In summary, warnings may be more effective in helping consumers identify unhealthful products high in energy, fat, sugar and sodium content in a supermarket environment than more detailed semi-directive FOP labels, such as the traffic-light system. Warnings can potentially discourage consumers from buying unhealthful products which have been linked with obesity and several noncommunicable diseases. Although there is evidence in the literature that health warnings can be a cost-effective policy to increase health knowledge and risk perception and can modify behaviour ${ }^{(53,54)}$, research is needed to evaluate if they can effectively modify consumers' food choices.

\section{Acknowledgements}

Financial support: The authors are indebted to Comisión Sectorial de Investigación Científica and Espacio Interdisciplinario (Universidad de la República) for financial support. The funders had no role in the design, analysis or writing of this article. Conflict of interest: None. Authorship: All authors contributed to the development of the research. G.A. and A.A. analysed the data. A.A., G.A., A.G. and M.R.C. wrote the paper. Ethics of human subject participation: This study was conducted according to the guidelines laid down in the Declaration of Helsinki and all procedures involving human subjects were approved by the Ethics Committee of the School of Chemistry of Universidad de la República (Uruguay). Written informed consent was obtained from all participants and formally recorded.

\section{References}

1. Monteiro CA, Levy RB, Claro RM et al. (2011) Increasing consumption of ultra-processed foods and likely impact on human health: evidence from Brazil. Public Health Nutr 14, 5-13.

2. Popkin BM, Adair LS \& Ng SW (2012) Global nutrition transition and the pandemic of obesity in developing countries. Nutr Rev 70, 3-21.

3. Moubarac J-C, Parra DC, Cannon G et al. (2014) Food classification systems based on food processing: significance and implications for policies and actions: a systematic literature review and assessment. Curr Obes Rep 3, 256-272.

4. Hawkes C, Smith TG, Jewell J et al. (2015) Smart policies for obesity prevention. Lancet 385, 2410-2421.

5. Capacci S, Mazzocchi M, Shankar B et al. (2012) Policies to promote healthy eating in Europe. A structured review of instruments and their effectiveness. Nutr Rev 70, 188-200.

6. Storcksdieck genannt Bonsmann S, Fernández Celemín L, Larrañga A et al. (2010) Penetration of nutrition information on food labels across the EU-27 plus Turkey. Eur J Clin Nutr 64, 1379-1385.

7. Cowburn G \& Stockley L (2005) Consumer understanding and use of nutrition labelling: a systematic review. Public Health Nutr 8, 21-28.

8. Graham DJ, Orquin JL \& Visschers VHM (2012) Eye tracking and nutrition label use: a review of the literature and recommendations for label enhancement. Food Policy 37, 378-382.

9. Sharf M, Sela R, Zentner G et al. (2012) Figuring out food labels. Young adults' understanding of nutritional information presented on food labels is inadequate. Appetite 58, 531-534.

10. Hawley KL, Roberto CA, Bragg MA et al. (2013) The science on front-of-package food labels. Public Health Nutr 16, 430-439.

11. Hodgkins C, Barnett J, Wasowicz-Kirylo G et al. (2012) Understanding how consumers categorise nutritional labels: a consumer derived typology for front-of-pack nutrition labelling. Appetite 59, 806-817.

12. Food Standards Agency (2007) Front-of-Pack Traffic Light Signpost Labelling. Technical Guidance. Issue 2. London: FSA. 
13. Larsson I, Lissner L \& Wilhelmsen L (1999) The 'Green keyhole' revisited: nutritional knowledge may influence food selection. Eur J Clin Nutr 53, 776-780.

14. Food Standards Australia New Zealand (2015) Guide for Industry to the Health Star Rating Calculator (HSRC). Canberra: FSANZ.

15. Lupton JR, Balantine DA, Black RM et al. (2010) The Smart Choices front-of-pack nutrition labelling program: rationale and development of nutrition criteria. Am J Clin Nutr 91, issue 4, 1078S-1089S.

16. Young L \& Swinburn B (2002) Impact of the Pick the Tick food information programme on the salt content of food in New Zealand. Health Promot Int 17, 13-19.

17. Antúnez L, Giménez A, Maiche A et al. (2015) Influence of interpretation aids on attentional capture, visual processing and understanding of front-of-pack nutrition labels. $J$ Nutr Educ Behav 47, 292-299.

18. Ares G, Giménez A, Bruzzone F et al. (2012) Attentional capture and understanding of nutrition labelling: a study based on response times. Int J Food Sci Nutr 63, 679-688.

19. Feunekes GIJ, Gortemaker IA, Willems AA et al. (2008) Front-of-pack nutrition labelling: testing effectiveness of different nutrition labelling formats front-of-pack in four European countries. Appetite 50, 57-70.

20. Roberto CA, Shivaram M, Martinez O et al. (2012) The Smart Choices front-of-package nutrition label. Influence on perceptions and intake of cereal. Appetite 58, 651-657.

21. Machín L, Giménez A, Curutchet MR et al. (2016) Motives underlying food choice for children and perception of nutritional information among low-income mothers in a Latin American country. J Nutr Educ Behav 48, 478-485.

22. Black A \& Rayner M (1992) Just Read the Label. London: The Stationery Office.

23. Corvalán C, Reyes M, Garmendia ML et al. (2013) Structural responses to the obesity and non-communicable disease epidemic: the Chilean law of food labelling and advertising. Obes Rev 14, 79-87.

24. Ministerio de Salud (2015) Decreto número 13, de 2015. Santiago: Ministerio de Salud.

25. Pan American Health Organization (2015) Ultra-Processed Food and Drink Products in Latin America: Trends, Impact on Obesity, Policy Implications. Washington, DC: PAHO.

26. Grunert KG \& Wills JM (2007) A review of European research on consumer response to nutrition information on food labels. J Public Health 15, 385-399.

27. Mackison D, Wrieden WL \& Anderson AS (2010) Validity and reliability testing of a short questionnaire to assess consumers' use, understanding and perception of food labels. Eur J Clin Nutr 64, 210-217.

28. Bialkova S \& van Trijp HCM (2011) An efficient methodology for assessing attention to and effect of nutrition information displayed front-of-pack. Food Qual Prefer 22, 592-601.

29. Becker MW, Sundar RP, Bello N et al. (2016) Assessing attentional prioritization of front-of-pack nutrition labels using change detection. Appl Ergon 54, 90-99.

30. Bix L, Prashant Sundar R, Bello NM et al. (2015) To see or not to see: do front of pack nutrition labels affect attention to overall nutrition information? PLoS One 10, e 0139732.

31. Aschemann-Witzel J, Grunert KG, van Trijp HCM et al. (2013) Effects of nutrition label format and product assortment on the healthfulness of food choice. Appetite 71, 63-74.

32. Maubach N, Hoek J \& Mather D (2014) Interpretive front-ofpack nutrition labels. Comparing competing recommendations. Appetite 82, 67-77.

33. van Herpen E, Hieke S \& van Trijp HCM (2014) Inferring product healthfulness from nutrition labelling. The influence of reference points. Appetite 72, 138-149.
34. Treisman AM \& Gelade G (1980) A feature-integration theory of attention. Cogn Psychol 12, 97-136.

35. Jarvis BG (2004) DirectRT Research Software, Version 2004. New York: Emprisoft.

36. R Core Team (2016) $R$ : A Language and Environment for Statistical Computing. Vienna: R Foundation for Statistical Computing.

37. van Herpen E \& van Trijp HCM (2011) Front-of-pack nutrition labels. Their effect on attention and choices when consumers have varying goals and time constraints. Appetite 57, 148-160.

38. Clement J (2007) Visual influence on in-store buying decisions: an eye-track experiment on the visual influence of packaging design. J Mark Manage 23, 917-928.

39. Orquin JL \& Mueller Loose S (2013) Attention and choice: a review on eye movements in decision making. Acta Psychol 144, 190-206.

40. Grunert KG, Fernández-Celemín L, Wills JM et al. (2010) Use and understanding of nutrition information on food labels in six European countries. J Public Health 18, 261-277.

41. Braun CC \& Silver NC (1995) Interaction of warning label features: determining the contributions of three warning characteristics. In Proceedings of the Human Factors and Ergonomics Society 39th Annual Meeting, pp. 984-988. Santa Monica, CA: Human Factors Society.

42. Chapanis A (1994) Hazards associated with three signal words and four colours on warning signs. Ergonomics 37, 265-275.

43. Machín L, Cabrer M, Curutchet MR et al. (2017) Healthfulness perception of ultra-processed products featuring different front-of-pack nutritional information schemes across two income levels. J Nutr Educ Behav 49, 330-338.e1.

44. Barone MJ, Rose RL, Manning KC et al. (1996) Another look at the impact of reference information on consumer impressions of nutrition information. J Public Policy Mark 15, 55-62.

45. Kelly B, Hughes C, Chapman K et al. (2009) Consumer testing of the acceptability and effectiveness of front-of-pack food labelling systems for the Australian grocery market. Health Promot Int 24, 120-129.

46. Visschers VHM \& Siegrist M (2009) Applying the evaluability principle to nutrition table information. How reference information changes people's perception of food products. Appetite 51, 505-512.

47. Soedeberg Miller LM, Cassady DL, Beckett LA et al. (2015) Misunderstanding of front-of-package nutrition information on US food products. PLoS One 10, e0125306.

48. Borgmeier I \& Westenhoefer J (2009) Impact of different food label formats on healthiness evaluation and food choice of consumers. A randomised-controlled study. $B M C$ Public Health 9, 184.

49. Watson WL, Kelly B, Hector D et al. (2014) Can front-ofpack labelling schemes guide healthier food choices? Australian shoppers' responses to seven labelling formats. Appetite 72, 90-97.

50. Dodds P, Wolfenden L, Chapman K et al. (2014) The effect of energy and traffic light labelling on parent and child fast food selection: a randomised controlled trial. Appetite 73, 23-30.

51. Sacks G, Rayner M \& Swinburn B (2009) Impact of front-ofpack 'traffic-light' nutrition labelling on consumer food purchases in the UK. Health Promot Int 24, 344-352.

52. Sacks G, Tikellis K, Millar L et al. (2011) Impact of 'traffic-light' nutrition information on online food purchases in Australia. Australian and New Zealand J Public Health 35, 122-126.

53. Hammond D (2011) Health warning messages on tobacco products: a review. Tob Control 20, 327-337.

54. Wilkinson C \& Room R (2009) Warnings on alcohol containers and advertisements: international experience and evidence on effects. Drug Alcohol Rev 28, 426-435. 